\title{
Rise of multidrug-resistant non-vaccine serotype 15A Streptococcus pneumoniae in the United Kingdom, 2001 to 2014
}

C Sheppard ${ }^{1}$, NK Fry ${ }^{1}$, S Mushtaq ${ }^{2}$, N Woodford ${ }^{2}$, R Reynolds ${ }^{45}$, R Janes ${ }^{6}$, R Pike ${ }^{2}$, R Hill ${ }^{2}, M_{\text {Kimuli }}^{2}$, P Staves ${ }^{2}, M$ Doumith ${ }^{2}$, T Harrison ${ }^{2}$, DM Livermore 2

1. Respiratory and Vaccine Preventable Bacteria Reference Unit, Public Health England, London, United Kingdom

2. Antimicrobial Resistance and Healthcare Associated Infections Reference Unit, Public Health England, London, United Kingdom

3. Norwich Medical School, University of East Anglia, Norwich, United Kingdom

4. Southmead Hospital, Bristol, United Kingdom

5. British Society for Antimicrobial Chemotherapy, Birmingham, United Kingdom

6. LGC, Fordham, United Kingdom

Correspondence: David Livermore (d.livermore@uea.ac.uk)

Citation style for this article:

Sheppard C, Fry NK, Mushtaq S, Woodford N, Reynolds R, Janes R, Pike R, Hill R, Kimuli M, Staves P, Doumith M, Harrison T, Livermore DM. Rise of multidrug resistant non-vaccine serotype 15A Streptococcus pneumoniae in the United Kingdom, 2001 to 2014. Euro Surveill. 2016;21(50):pii=30423. DOI: http://dx.doi. org/10.2807/1560-7917.ES.2016.21.50.30423

Article submitted on 19 March 2016 / accepted on 17 August 2016 / published on 15 December 2016

Conjugate vaccines have reduced pneumococcal disease in vaccinated children and unvaccinated adults, but non-vaccine serotypes are of concern, particularly if antibiotic resistant. We reviewed Streptococcus pneumoniae collected via: (i) the British Society for Antimicrobial Chemotherapy (BSAC) surveillances from 2001-2014; (ii) Public Health England's (PHE) invasive isolate surveillance from 2005-2014 and (iii) referral to PHE for resistance investigation from 2005-2014. Serotype $15 \mathrm{~A}$ increased in all series, with many representatives showing triple resistance to macrolides, tetracyclines and penicillin. 15A was consistently among the 10 most prevalent serotypes from 2011 in PHE and BSAC invasive isolate/bacteraemia surveillance but never previously; $26-33 \%$ of these invasive $15 \mathrm{~A}$ isolates had triple resistance. BSAC respiratory isolates were only serotyped in 2013/14 and 2014/15 (October to September); $15 \mathrm{~A}$ was most prevalent serotype in both periods, comprising $9-11 \%$ of isolates, $38-48 \%$ of them with triple resistance. Serotype $15 \mathrm{~A}$ represented $0-4 \%$ of $S$. pneumoniae referred to $\mathrm{PHE}$ for reference investigation annually until 2008 but rose to $29 \%$ (2013) and $32 \%$ (2014). Almost all multidrug-resistant $15 \mathrm{~A}$ isolates were sequence type (ST) 63 variants, whereas susceptible $15 \mathrm{~A}$ isolates were clonally diverse. The rise of serotype $15 \mathrm{~A}$ suggests that pneumococcal conjugate vaccines will need ongoing adaptation.

\section{Introduction}

Seven-valent pneumococcal conjugate vaccine (Prevenar 7, PCV7) first became available internationally in 2000, and protects against invasive Streptococcus pneumoniae infection by serotypes $4,6 \mathrm{~B}, 9 \mathrm{~V}, 14,18 \mathrm{C}$,
$19 \mathrm{~F}$ and $23 \mathrm{~F}$. Numerous countries have reported that deployment reduced the incidence of invasive (i.e. blood and cerebrospinal fluid (CSF)) S. pneumoniae disease both in children, who are vaccinated, and in elderly adults, who benefit through reduced carriage and transmission of virulent serotypes by children [1-4]. Antibiotic resistance was historically concentrated in five PCV7-targeted serotypes (6B, 9V, 14, 19F and 23F) [5] and several countries have reported reductions in the prevalence of resistance as these were displaced [6]. United Kingdom (UK) experience conforms to these general patterns [7], with the caveat that penicillin-nonsusceptible $S$. pneumoniae were uncommon before the vaccine's introduction to the childhood schedule in 2006/07, meaning that little further fall occurred; macrolide resistance was reduced, reflecting displacement of a resistant serotype 14 lineage $[8,9]$.

The success of $\mathrm{PCV}_{7}$ was partly offset by rises in other serotypes; notably $19 \mathrm{~A}$, where multidrug resistance to antibiotics became frequent $[10,11]$. This was countered by replacing $\mathrm{PCV}_{7}$ with a 13 -valent conjugate vaccine (PCV13), additionally covering serotypes 1, 3, 5, 6A, 7F and $19 \mathrm{~A}$. PCV13 replaced $\mathrm{PCV}_{7}$ in the UK in April 2010 and this switch was followed by (i) reduced infant carriage of these additional serotypes [12], and (ii) a further $56 \%$ reduction in invasive disease incidence from a post-PCV 7 baseline [13]. Again, however, rises are being seen in other, non-vaccine, serotypes, principally $8,10 \mathrm{~A}, 12 \mathrm{~F}, 15 \mathrm{~A}$ and $24 \mathrm{~F}$ [13]. Serotype $15 \mathrm{~A}$ is of particular interest since multidrug-resistant isolates belonging to this serotype have been reported as far apart as east Asia [14-16], North America [17,18], Norway [19], Italy [20] and Australia [21]. Here, we explore the rise 
TABLE 1

Ten most-represented pneumococcal serotypes in the British Society for Antimicrobial Chemotherapy bacteraemia surveillance, United Kingdom and Republic of Ireland, 2001-2014 ( $\mathrm{n}=3,206$ isolates)

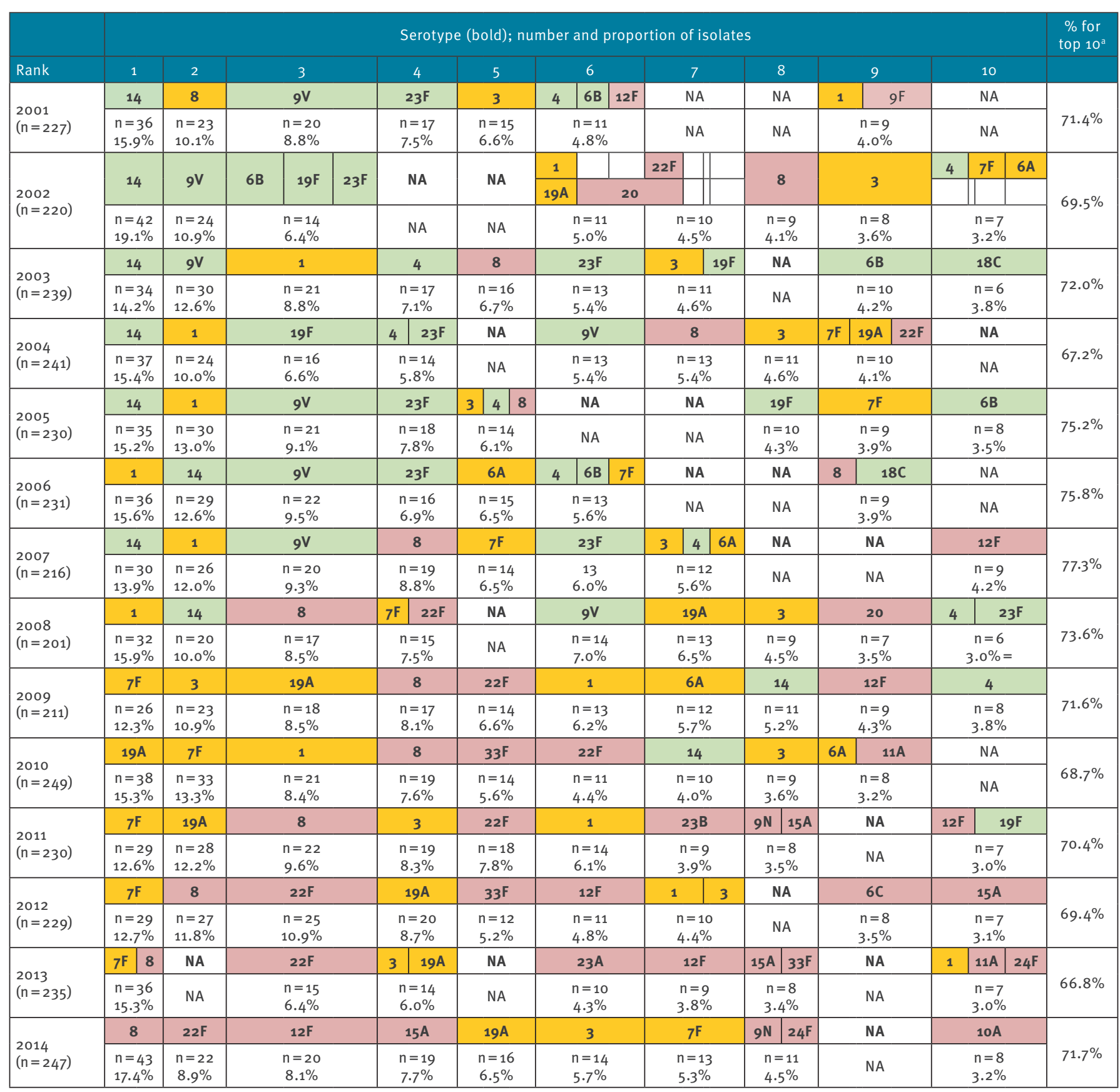

NA: not applicable.

Green: covered by PCV7; yellow: additional types covered by PCV13; pink: not covered by any conjugate vaccine.

a When there is a tie for tenth rank, only one of the tied serotypes is counted into the percentage total for the top 10. 
of serotype 15A S. pneumoniae in the UK and Ireland at epidemiological and molecular levels, using data from both the British Society for Antimicrobial Chemotherapy (BSAC) and Public Health England (PHE) surveillances.

\section{Methods}

\section{British Society for Antimicrobial \\ Chemotherapy surveillance}

The BSAC Bacteraemia and Respiratory Surveillance Programmes have been described previously [22-24]. Both collect isolates from across the UK and Republic of Ireland. The Bacteraemia programme runs on the calendar year. Until 2009 we asked participating laboratories to send up to 10 consecutive bloodstream S. pneumoniae isolates per annum from each of 25 hospital laboratories; from 2010 we have similarly sought seven consecutive bloodstream isolates per annum from each of 40 hospital laboratories. Isolates have been serotyped throughout, and results were reviewed across the years 2001 to 2014, inclusive. The Respiratory Programme runs on an October-September year, designated e.g. 2013/14, so that isolates from each winter peak of respiratory disease are not split between calendar years. It examines consecutive isolates from lower respiratory tract infections (LRTIS) in non-hospitalised patients or those hospitalised forless than 48 hours. Until 2013/14 the BSAC Respiratory Surveillance Programme did not routinely serotype isolates, therefore only 2013/14 and 2014/15 data were reviewed. In both these years the surveillance sought 14 consecutive LRTI S. pneumoniae isolates from each of the same 40 laboratories contributing to the Bacteraemia surveillance. Actual numbers of isolates collected in both surveillances were somewhat below these targets (see Results) and, in most years, one or two recruited laboratories failed to collect, and were subsequently dropped and replaced by alternative sites. Hospital laboratory mergers, mostly in the past 5 years, have also meant that participating microbiology laboratories increasingly source isolates from multiple hospitals, augmenting representativeness.

\section{Public Health England invasive isolate surveillance}

PHE Colindale routinely seeks submission of all invasive (i.e. blood and CSF) S. pneumoniae from hospital laboratories in England, Wales and Northern Ireland, receiving ca 4,000-5,000 isolates each year), over $95 \%$ of them from blood. Results of this surveillance were reviewed from 2005 (i.e. 1 year before $\mathrm{PCV}_{7}$ was introduced) to 2014 . Susceptibility testing is performed on a subset of these isolates, comprising all those from laboratories contributing to European Antimicrobial Resistance Surveillance (EARS)-net [25]; this total fluctuated between 1,159 and 2,066 organisms annually over the study period.

\section{Public Health England reference laboratory} submissions

Besides surveillance isolates from invasive infections, PHE receives variable numbers of $S$. pneumoniae as reference submissions from respiratory and other nonsterile sites, principally eye and ear infections. Most are sent for investigation because the sender perceives them to have unusual resistance patterns, although senders' definitions of unusual vary and may be contingent on the site of the infection. Over $95 \%$ of isolates are from laboratories in England, Wales and Northern Ireland, with the remaining ca $5 \%$ largely from Scotland and the Republic of Ireland. Data were reviewed across the period 2005 to 2014 .

\section{Identification, serotyping and susceptibility testing}

All surveillance and referred isolates were confirmed as forming alpha-haemolytic colonies on horse blood agar and being inhibited by a $5 \mu \mathrm{g}$ optochin (ethylhydrocupreine hydrochloride) disc (Oxoid-Thermofisher, Basingstoke, UK). Isolates with atypical colonial morphology, or which could not be serotyped (below), were confirmed as being lysed within $30 \mathrm{~min}$ by $2 \%$ sodium deoxycholate, and being catalase-negative when tested with $3 \%$ hydrogen peroxide. For serotyping, isolates were grown overnight in Todd Hewitt broth at $35^{\circ} \mathrm{C}$ with $5 \% \mathrm{CO}_{2}$, harvested by centrifugation at $453 \mathrm{~g}$ for $30 \mathrm{~min}$, then re-suspended in a small residual volume of broth and subjected to slide agglutination tests with standard antisera (Statens Serum Institut, Copenhagen, Denmark) [26]. Agar dilution susceptibility tests were performed in accordance with BSAC guidelines [27], using IsoSensitest agar (OxoidThermofisher) supplemented with $5 \%$ defibrinated horse blood and incubated at $35-37^{\circ} \mathrm{C}$ in a $5 \% \mathrm{CO}_{2}$ atmosphere. 'Triple resistance' was defined as resistant to erythromycin (minimum inhibitory concentration $(\mathrm{MIC})>0.5 \mathrm{mg} / \mathrm{L}$ ) and tetracycline $(\mathrm{MIC}>2 \mathrm{mg} / \mathrm{L})$, and non-susceptible to penicillin (MIC>0.06 mg/L), based on EUCAST breakpoints [28])

\section{DNA extraction, sequencing and bioinformatic analysis}

Isolates were grown on horse blood agar (PHE Media Services) and treated by the Qiagen-recommended method for lysis of Gram-negative bacteria (Qiagen, Manchester, UK), which is effective for S. pneumoniae and simpler than the Gram-positive protocol. DNA was extracted from the lysates using a QIAsymphony SP automated instrument (Qiagen) and a QIAsymphony DSP DNA Mini Kit, using a tissue extraction protocol. DNA concentrations were measured using the Quant-IT Broad Range DNA Kit (Life Technologies, Paisley, UK) and GloMax 96 Microplate Luminometer (Promega, Southampton, UK). After adjusting to a concentration of $10-30 \mathrm{ng} / \mu \mathrm{l}$, DNA was sent for whole genome sequencing (WGS) by Illumina methodology. The resulting data were automatically analysed using a bespoke bioinformatic pipeline for $S$. pneumoniae, developed by PHE. Among other things, this (i) checks species 
TABLE 2

Major serotypes and associations with resistance among Streptococcus pneumoniae from the British Society for Antimicrobial Chemotherapy Respiratory Surveillance, United Kingdom and Republic of Ireland, 2013/14 and 2014/15 $(\mathrm{n}=805)$

\begin{tabular}{|c|c|c|c|c|c|c|}
\hline Serotype & & October 2013 to Sep & r 2014 & & October 2014 to S & ber 2015 \\
\hline & Count & $\%$ of total isolates & $\begin{array}{c}\text { No (\%) with } \\
\text { triple resistance }\end{array}$ & Count & $\%$ of total isolates & $\begin{array}{c}\text { No (\%) with } \\
\text { triple resistance }\end{array}$ \\
\hline $15 \mathrm{~A}$ & 34 & 9.1 & $13(38.2 \%)$ & 46 & 10.7 & $22(47.8 \%)$ \\
\hline $23 \mathrm{~B}$ & 26 & 6.9 & $1(3.8 \%)$ & 21 & 4.9 & 0 \\
\hline 3 & 22 & 5.9 & 0 & 26 & 6.0 & 0 \\
\hline $11 \mathrm{~A}$ & 21 & 5.6 & $1(4.8 \%)$ & 34 & 7.9 & $1(2.9 \%)$ \\
\hline $23 \mathrm{~A}$ & 21 & 5.6 & 0 & 30 & 7.0 & $4(13.3 \%)$ \\
\hline $22 \mathrm{~F}$ & 19 & 5.1 & 0 & 17 & 4.0 & 0 \\
\hline $6 C$ & 18 & 4.8 & 0 & 12 & 2.8 & 0 \\
\hline $19 \mathrm{~A}$ & 17 & 4.5 & $5(29.4 \%)$ & 14 & 3.3 & $4(28.6 \%)$ \\
\hline $24 \mathrm{~F}$ & 16 & $4 \cdot 3$ & 0 & 12 & 2.8 & $1(8.3 \%)$ \\
\hline $35 \mathrm{~F}$ & 14 & 3.7 & 0 & 14 & 3.3 & 0 \\
\hline $10 \mathrm{~A}$ & 14 & 3.7 & 0 & 12 & 2.8 & 0 \\
\hline 31 & 14 & 3.7 & 0 & 16 & 3.7 & 0 \\
\hline $16 \mathrm{~F}$ & 12 & 3.2 & $1(8.3 \%)$ & 19 & 4.4 & 0 \\
\hline $15 B$ & 11 & 2.9 & 0 & 3 & 0.7 & 0 \\
\hline $17 \mathrm{~F}$ & 11 & 2.9 & 0 & 16 & 3.7 & 0 \\
\hline $19 \mathrm{~F}$ & 11 & 2.9 & $3(27.3 \%)$ & 14 & 3.3 & $5(35.7 \%)$ \\
\hline $35 \mathrm{~B}$ & 11 & 2.9 & 0 & 18 & 4.2 & 0 \\
\hline 8 & 10 & 2.7 & 0 & 12 & 2.8 & $1(8.3 \%)$ \\
\hline $\begin{array}{l}\text { Other } \\
\text { serotypes, } \\
\text { with }<10 \text { isolates } \\
\text { in one or both } \\
\text { years }\end{array}$ & 73 & 19.4 & $3(4.9 \%)^{a}$ & 85 & $(21.7)$ & $10(2.3 \%)^{\mathrm{a}}$ \\
\hline $\mathrm{PCV}_{7}$ serotypes & 17 & 4.5 & NA & 20 & 4.6 & NA \\
\hline PCV13 serotypes & 63 & 16.8 & NA & 67 & 15.6 & NA \\
\hline Total & 375 & 100 & $27(7.2 \%)$ & 430 & 100 & 49 (11.4\%) \\
\hline
\end{tabular}

NA: not applicable; PCV: pneumococcal conjugate vaccine.

${ }^{a}$ In $2013 / 14$, three 6B isolates had triple resistance; the 10 'Other serotype' isolates with triple resistance in $2014 / 15$ comprised three nontypeable, two $12 \mathrm{~F}$ and single representatives of $6 \mathrm{~B}, 7 \mathrm{~F}, 9 \mathrm{~N} 9 \mathrm{~V}$ and 23 .

identification by a kmer method and (ii) automatically assigns MLST sequence types (STs), identified by mapping the reads against all $S$. pneumoniae allele variants held in the MLST database [29], using a modification of the short-read sequence typing (SRST) software [30]. Resistance genes affecting susceptibility for macrolides and tetracyclines were identified, and their sequences reviewed.

\section{Results}

Serotype trends, British Society for Antimicrobial Chemotherapy bacteraemia surveillance

Prior to widespread UK deployment of $\mathrm{PCV}_{7}$ in the 2006/07 season, S. pneumoniae belonging to its target serotypes accounted for around half (44.4-53.6\% in each of the years 2001 to 2006 inclusive) of all the S. pneumoniae collected in the BSAC bacteraemia surveillance but these declined to $4.7 \%$ of isolates by 2013 and $2.0 \%$ in 2014 . Serotype 14 was the most common type in 6 of the 7 years from 2001 to 2007, comprising $13-20 \%$ of all isolates (Table 1 ) and accounting for $61 \%$ of all erythromycin-resistant isolates. By 2013, however, serotype 14 had only a single representative (0.4\%), and none in 2014. Other serotypes became relatively more frequent as the $\mathrm{PCV}_{7}$ types declined, notably $7 \mathrm{~F}$ and $19 \mathrm{~A}$, whereas serotype 1 had been expanding since 2001. These three types are within the spectrum of $\mathrm{PCV}_{13}$ and have declined, with variable rapidity, following its replacement of $\mathrm{PCV}_{7}$ in 2010 . A further PCV13 type, serotypes 3, shows much less evidence of decline, as also noted elsewhere [13].

Serotype $15 \mathrm{~A}$ isolates were encountered in each year from 2010 and the serotype was in the top 10 from 2011 onwards, whereas previously the type was sporadic. Other types that had long been encountered at moderate to low prevalence also became more prominent, 
Predominant serotypes among S. pneumoniae serotyped by the Respiratory and Vaccine Preventable Bacteria Reference Unit, Public Health England from invasive infections, 2005-2014 ( $\mathrm{n}=45,645)$

\begin{tabular}{|c|c|c|c|c|c|c|c|c|c|c|c|}
\hline \multirow[b]{2}{*}{ Rank } & \multicolumn{10}{|c|}{ Serotype (bold) number and proportion of isolates } & \multirow{2}{*}{$\begin{array}{l}\text { Proportion for top } \\
10\end{array}$} \\
\hline & 1 & 2 & 3 & 4 & 5 & 6 & 7 & 8 & 9 & 10 & \\
\hline \multirow{3}{*}{$\begin{array}{l}2005 \\
n=4,662\end{array}$} & 14 & 1 & 8 & $9 \mathrm{~V}$ & 4 & $23 \mathrm{~F}$ & 3 & 6B & $7 \mathrm{~F}$ & $19 \mathrm{~F}$ & \multirow{3}{*}{$73.3 \%$} \\
\hline & 701 & 528 & 357 & 333 & 327 & 271 & 250 & 248 & 208 & 195 & \\
\hline & $15.0 \%$ & $11.3 \%$ & $7.7 \%$ & $7.1 \%$ & $7.0 \%$ & $5.8 \%$ & $5.4 \%$ & $5.3 \%$ & $4.5 \%$ & $4.2 \%$ & \\
\hline \multirow{3}{*}{$\begin{array}{l}2006 \\
n=4,857\end{array}$} & 14 & 1 & $9 \mathrm{~V}$ & 8 & $23 \mathrm{~F}$ & 4 & 3 & 6B & $7 F$ & $19 \mathrm{~F}$ & \multirow{3}{*}{$72.1 \%$} \\
\hline & 660 & 611 & 337 & 321 & 300 & 288 & 268 & 256 & 249 & 210 & \\
\hline & $13.6 \%$ & $12.6 \%$ & $6.9 \%$ & $6.6 \%$ & $6.2 \%$ & $5.9 \%$ & $5.5 \%$ & $5.3 \%$ & $5.1 \%$ & $4.3 \%$ & \\
\hline \multirow{3}{*}{$\begin{array}{l}2007 \\
n=4,673\end{array}$} & 1 & 14 & $9 \mathrm{~V}$ & 8 & $7 F$ & 3 & 4 & $6 \mathrm{~A}$ & $23 \mathrm{~F}$ & 6B & \multirow{3}{*}{$69.1 \%$} \\
\hline & 583 & 449 & 351 & 348 & 316 & 278 & 238 & 237 & 231 & 197 & \\
\hline & $12.5 \%$ & $9.6 \%$ & $7.5 \%$ & $7.4 \%$ & $6.8 \%$ & $5.9 \%$ & $5.1 \%$ & $5.1 \%$ & $4.9 \%$ & $4.2 \%$ & \\
\hline \multirow{3}{*}{$\begin{array}{l}2008 \\
n=4,978\end{array}$} & 1 & $7 F$ & 8 & 3 & $22 \mathrm{~F}$ & $19 A$ & $6 A$ & 14 & $9 \mathrm{~V}$ & $23 \mathrm{~F}$ & \multirow{3}{*}{$66.4 \%$} \\
\hline & 592 & 474 & 372 & 359 & 328 & 307 & 239 & 238 & 206 & 189 & \\
\hline & $11.9 \%$ & $9.5 \%$ & $7.5 \%$ & $7.2 \%$ & $6.6 \%$ & $6.2 \%$ & $4.8 \%$ & $4.8 \%$ & $4.1 \%$ & $3.8 \%$ & \\
\hline \multirow{3}{*}{$\begin{array}{l}2009 \\
n=5,000\end{array}$} & $7 F$ & 1 & $19 A$ & 3 & $22 \mathrm{~F}$ & 8 & $6 A$ & $12 \mathrm{~F}$ & 14 & $33 \mathrm{~F}$ & \multirow{3}{*}{$67.7 \%$} \\
\hline & 553 & 501 & 490 & 438 & 423 & 393 & 189 & 148 & 131 & 118 & \\
\hline & $11.1 \%$ & $10.0 \%$ & $9.8 \%$ & $8.8 \%$ & $8.5 \%$ & $7.9 \%$ & $3.8 \%$ & $3.0 \%$ & $2.6 \%$ & $2.4 \%$ & \\
\hline \multirow{3}{*}{$\begin{array}{l}2010 \\
n=4,881\end{array}$} & $7 \mathrm{~F}$ & $19 A$ & 1 & 3 & 8 & $22 \mathrm{~F}$ & $33 \mathrm{~F}$ & $6 C$ & $12 \mathrm{~F}$ & $11 \mathrm{~A}$ & \multirow{3}{*}{$69.9 \%$} \\
\hline & 675 & 640 & 445 & 362 & 362 & 361 & 164 & 161 & 139 & 102 & \\
\hline & $13.8 \%$ & $13.1 \%$ & $9.1 \%$ & $7.4 \%$ & $7.4 \%$ & $7.4 \%$ & $3.4 \%$ & $3.3 \%$ & $2.8 \%$ & $2.1 \%$ & \\
\hline \multirow{3}{*}{$\begin{array}{l}2011 \\
n=4,549\end{array}$} & $7 F$ & $19 \mathrm{~A}$ & 8 & 1 & 3 & $22 \mathrm{~F}$ & $12 \mathrm{~F}$ & $33 \mathrm{~F}$ & $6 C$ & $15 \mathrm{~A}$ & \multirow{3}{*}{$71.8 \%$} \\
\hline & 665 & 538 & 424 & 391 & 382 & 348 & 139 & 131 & 126 & 124 & \\
\hline & $14.6 \%$ & $11.8 \%$ & $9.3 \%$ & $8.6 \%$ & $8.4 \%$ & $7.7 \%$ & $3.1 \%$ & $2.9 \%$ & $2.8 \%$ & $2.7 \%$ & \\
\hline \multirow{3}{*}{$\begin{array}{l}2012 \\
n=4,092\end{array}$} & $7 F$ & 8 & $19 A$ & $22 \mathrm{~F}$ & 3 & 1 & $15 A$ & $33 \mathrm{~F}$ & $6 C$ & $12 \mathrm{~F}$ & \multirow{3}{*}{$68.2 \%$} \\
\hline & 485 & 456 & 369 & 357 & 276 & 243 & 176 & 155 & 148 & 125 & \\
\hline & $11.9 \%$ & $11.1 \%$ & $9.0 \%$ & $8.7 \%$ & $6.7 \%$ & $5.9 \%$ & $4.3 \%$ & $3.8 \%$ & $3.6 \%$ & $3.1 \%$ & \\
\hline \multirow{3}{*}{$\begin{array}{l}2013 \\
n=3,995\end{array}$} & 8 & $7 F$ & $22 \mathrm{~F}$ & $19 \mathrm{~A}$ & 3 & $15 \mathrm{~A}$ & $12 \mathrm{~F}$ & 1 & $24 \mathrm{~F}$ & $33 \mathrm{~F}$ & \multirow{3}{*}{$66.4 \%$} \\
\hline & 545 & 415 & 320 & 293 & 274 & 203 & 174 & 153 & 141 & 134 & \\
\hline & $13.6 \%$ & $10.4 \%$ & $8.0 \%$ & $7.3 \%$ & $6.9 \%$ & $5.1 \%$ & $4.4 \%$ & $3.8 \%$ & $3.5 \%$ & $3.4 \%$ & \\
\hline \multirow{3}{*}{$\begin{array}{l}2014 \\
n=3,959\end{array}$} & 8 & $12 \mathrm{~F}$ & $22 \mathrm{~F}$ & 3 & $19 \mathrm{~A}$ & $15 \mathrm{~A}$ & $7 F$ & $9 \mathrm{~N}$ & $33 \mathrm{~F}$ & $24 \mathrm{~F}$ & \multirow{3}{*}{$67.9 \%$} \\
\hline & 599 & 336 & 334 & 243 & 229 & 224 & 219 & 170 & 168 & 167 & \\
\hline & $15.1 \%$ & $8.5 \%$ & $8.4 \%$ & $6.1 \%$ & $5.8 \%$ & $5.7 \%$ & $5.5 \%$ & $4.3 \%$ & $4.2 \%$ & $4.2 \%$ & \\
\hline
\end{tabular}

PCV: pneumococcal conjugate vaccine.

Green: covered by PCV7.

Yellow: additional types covered by PCV13.

Pink: not covered by any conjugate vaccine.

99\% of isolates are from England, Wales and Northern Ireland, with the remaining few from Scotland, Crown Dependencies, Republic of Ireland and elsewhere.

including serotypes 8 , and (albeit with considerable year-on-year variation) $22 \mathrm{~F}$.

Triple resistance was seen in just 6o/3,206 isolates $(1.97 \%)$ throughout the period reviewed and its prevalence exceeded $10 \%$ only among isolates of serotypes 37 (2/3 isolates), 6B (13/90 isolates, $14.4 \%)$ and, most strikingly, $15 \mathrm{~A}$ (13/50, 26.0\%). Triple-resistant serotype $15 A$ S. pneumoniae were received in every year from 2011, although never previously. This observation, along with increasing numbers of $15 \mathrm{~A}$ isolates among PHE reference submissions (below), prompted the present analysis.
Serotypes among British Society for Antimicrobial Chemotherapy respiratory isolates

Unlike those collected in the BSAC Bacteraemia Surveillance, S. pneumoniae from the BSAC Respiratory Surveillance were not routinely serotyped until 2013/14, when $15 \mathrm{~A}$ proved to be the most frequent serotype (Table 2), comprising $349.1 \%$ of all 375 isolates collected, with a similar pattern in 2014/15, when $15 \mathrm{~A}$ comprised $46 / 430$ (10.7\%) of isolates. What is more, $15 \mathrm{~A}$ was one of only four serotypes (the others being $6 \mathrm{~B}, 19 \mathrm{~A}$ and $19 \mathrm{~F}$ ) where triple resistance was seen in over $10 \%$ of representatives. Overall, triple resistance was seen in $13 / 34(38.2 \%)$ serotype $15 \mathrm{~A}$ isolates vs 


\section{TABLE 4}

Proportions of isolates with triple resistance to penicillin, erythromycin and tetracycline among frequent serotypes of Streptococcus pneumoniae from blood and cerebrospinal fluid infections, Public Health England surveillance, 2005-2014 $(\mathrm{n}=13,551)$

\begin{tabular}{|l|c|c|c|}
\hline Serotype & Total & Triple resistance & \% Triple resistance \\
\hline $15 \mathrm{~A}$ & 330 & 104 & 31.5 \\
\hline $6 \mathrm{~B}$ & 420 & 51 & 12.1 \\
\hline $19 \mathrm{~F}$ & 401 & 45 & 11.2 \\
\hline $19 \mathrm{~A}$ & 987 & 83 & 8.4 \\
\hline $23 \mathrm{~F}$ & 360 & 15 & 4.2 \\
\hline $24 \mathrm{~F}$ & 124 & 5 & 4.0 \\
\hline $9 \mathrm{~V}$ & 562 & 19 & 3.4 \\
\hline 14 & 1,145 & 27 & 2.4 \\
\hline $6 \mathrm{~A}$ & 366 & 3 & 0.8 \\
\hline 8 & 1,197 & 3 & 0.3 \\
\hline $6 \mathrm{C}$ & 205 & 2 & 1.0 \\
\hline $9 \mathrm{~N}$ & 261 & 1 & 0.4 \\
\hline 3 & 777 & 2 & 0.3 \\
\hline $33 \mathrm{~F}$ & 239 & 2 & 0.8 \\
\hline 1 & 1,195 & 1 & 0.1 \\
\hline $22 \mathrm{~F}$ & 761 & 1 & 0.1 \\
\hline $12 \mathrm{~F}$ & 474 & 1 & 0.2 \\
\hline 4 & 334 & 0 & 0 \\
\hline $7 \mathrm{~F}$ & 1,155 & 0 & 0 \\
\hline All others & 2,258 & 0 & 3.5 \\
\hline $\begin{array}{l}\text { All isolates } \\
\text { and serotypes }\end{array}$ & 13,551 & 469 & \\
\hline & & & 0 \\
\hline & 159 & 2.5 \\
\hline
\end{tabular}

$99 \%$ of isolates are from England, Wales and Northern Ireland, with the remaining few from Scotland, Crown Dependencies, Republic of Ireland and elsewhere.

Serotypes that reached a top-10 ranking in any surveillance year in Table 3 are line-listed.

$14 / 341(4.1 \%)$ of all other isolates in 2013/14 ( $p<0.001$, logistic regression adjusted for clustering by centre); there was an even sharper difference, 24/46 (52.2\%) vs $25 / 384(6.5 \%)$ ( $p<0.001)$, in $2014 / 15$.

Also notable was the fact that $\mathrm{PCV}_{7}$ serotypes accounted for only $17 / 375$ (4.5\%) of all the respiratory S. pneumoniae in $2013 / 14$ and PCV13 types for just $63 / 375$ (16.8\%); corresponding figures in $2014 / 15$ were $18 / 430$ (4.3\%) for PCV7 types and 68/430 (15.8\%) for PCV13 types. The sole previous season when S. pneumoniae from the Respiratory Programme were typed was 2005/06, immediately before UK introduction of $\mathrm{PCV}_{7}$ [24]. Then, among 749 isolates, 312 (41.7\%) belonged to $\mathrm{PCV}_{7}$ types and 450 (60.1\%) to PCV13 types (assuming all serogroup 7 isolates belonged to serotype $7 \mathrm{~F}$ ) whereas 36 (4.8\%) belonged to serogroup 15, which was not split to its component (15A/B/C/F) serotypes. The declines in $\mathrm{PCV}_{7}$ types, $\mathrm{PCV}_{13}$ types, and the rise in serotype $15 \mathrm{~A}$ (compared with all serotype 15 in 2005/06) were all highly significant ( $p<0.001$, logistic regression adjusted for clustering by centre).
Serotype trends, Public Health England invasive isolate surveillance

PHE surveillance of invasive $S$. pneumoniae indicated dramatic changes in serotype prevalence over time, as reviewed previously $[7,13]$, with these paralleling the shifts seen for BSAC bacteraemia isolates. Specifically, in each of the years up to and including 2007 , five or six of the top 10 serotypes were $\mathrm{PCV}_{7}$ types, with serotype 14 the most abundant (Table 3), as in the BSAC series (Table 1). After 2007, PCV7 types declined, with none in the top 10 after 2009. Several of the additional types covered by $\mathrm{PCV}_{13}$, notably $3,6 \mathrm{~A}, 7 \mathrm{~F}$ and $19 \mathrm{~A}$, became relatively more prominent from 2006 until 2011 while serotype 1, also a PCV13 additional type, was prominent even before 2011. Except for serotype 3, which showed little convincing trend, these additional PCV13 types declined in rank after 2010/11, with the peaking of serotypes 1 and 19A being especially marked.

As in the BSAC series, serotype $15 A$ first appeared in the top 10 in 2011. It then advanced to seventh rank by 2012 and sixth rank in both 2013 and 2014, accounting for $5.7 \%$ of isolates $(224 / 3,959)$ in the latter year. Again, the proportion of resistance was striking: among the 330 tested, fully 104 (31.5\%) of bloodstream $15 \mathrm{~A}$ S. pneumoniae for all years pooled had triple resistance, whereas triple resistance rates for all other isolates that ever featured in the top 10 were under $12.5 \%$ (Table 4). Proportions of serotype $15 \mathrm{~A}$ isolates, taking 2005-2014 pooled, rose with the patient's age, from $1.3 \%$ in the $0-5$ year age group to $1.4 \%$ in the $6-35$ year age group, $0.6 \%$ in the $36-45$ year age group, $1.7 \%$ in the $46-55,56-65$ and $66-75$ year age groups, reaching $2.4 \%$ in the $76-85$ year age group and $3.1 \%$ among the over- 85 -year-olds $(p<0.001)$. Triple resistance was represented among serotype $15 \mathrm{~A} S$. pneumoniae throughout the surveillance period reviewed, with proportions as follows: $2005,0 / 3$ isolates with triple resistance; 2006, 1/4; 2007, 2/10; 2008, 4/13; 2009 7/13; 2010, $18 / 34 ; 2011,10 / 33 ; 2012,15 / 50 ; 2013,19 / 63$ and 2014 , $33 / 114$.

The isolates tested for antibiotic susceptibility and resistance $(n=13,551$, annual range 1,159-2,966 p.a.) are a subset of those in Table 3 and comprise all isolates from hospitals that participate in the EARS-net surveillance along with those bloodstream isolates where the referring laboratory specifically sought susceptibility testing. Inclusion of the latter group may over-represent resistant organisms, although there is no reason why it should do so disproportionately within particular serotypes.

Serotype trends, isolates referred to Public Health England for investigation of resistance Between 2005 and 2014, 1,536 S. pneumoniae from respiratory, ear and eye infections were referred to $\mathrm{PHE}$ (Table 5) for investigation of unusual resistance. These submissions constitute a heavily biased sample and lack a denominator, but do provide a rolling snapshot of $S$. pneumoniae isolates that sending laboratories 
Predominant serotypes among respiratory, ear and eye isolates of Streptococcus pneumoniae received by the Public Health England Colindale reference service, 2005-2014 $(n=1,536)$

\begin{tabular}{|c|c|c|c|c|c|c|c|c|c|c|c|c|c|}
\hline & \multicolumn{10}{|c|}{ Number of isolates of indicated serotype in year: } & \multirow[b]{2}{*}{ Grand total } & \multirow{2}{*}{$\begin{array}{l}\text { No with triple } \\
\text { resistance }\end{array}$} & \multirow{2}{*}{$\begin{array}{l}\% \text { with triple } \\
\text { resistance }\end{array}$} \\
\hline & 2005 & 2006 & 2007 & 2008 & 2009 & 2010 & 2011 & 2012 & 2013 & 2014 & & & \\
\hline Serotype $19 \mathrm{~F}$ & 15 & 31 & 41 & 50 & 55 & 35 & 23 & 14 & 12 & 14 & 290 & 232 & 80.0 \\
\hline Serotype 19A & 2 & 10 & 15 & 27 & 45 & 44 & 28 & 17 & 8 & 17 & 213 & 169 & 79.3 \\
\hline Serotype $15 A$ & 0 & 4 & 3 & 8 & 23 & 22 & 17 & 26 & 31 & 31 & 165 & 137 & 83.0 \\
\hline Serotype 6B & 5 & 18 & 39 & 35 & 25 & 13 & 4 & 7 & 3 & 2 & 151 & 104 & 68.9 \\
\hline Non-typeable rough & 3 & 8 & 16 & 23 & 33 & 18 & 10 & 15 & 5 & 2 & 133 & 81 & 60.9 \\
\hline Serotype $9 \mathrm{~V}$ & 7 & 36 & 25 & 15 & 18 & 10 & 0 & 0 & 1 & 2 & 114 & 16 & 14.0 \\
\hline Serotype 14 & 9 & 18 & 21 & 6 & 11 & 11 & 4 & 2 & 3 & 1 & 86 & 30 & 34.9 \\
\hline Serotype $23 \mathrm{~F}$ & 7 & 9 & 6 & 16 & 16 & 9 & 3 & 2 & 1 & 3 & 72 & 35 & 48.6 \\
\hline Serotype 35 B & 1 & 3 & 4 & 9 & 7 & 6 & 9 & 4 & 7 & 4 & 54 & 9 & 16.7 \\
\hline No serotype data & 1 & 2 & 0 & 0 & 0 & 6 & 1 & 1 & 5 & 26 & 42 & 22 & 52.4 \\
\hline Serotype $6 \mathrm{~A}$ & 0 & 3 & 5 & 5 & 3 & 6 & 1 & 2 & 0 & 1 & 26 & 7 & 26.9 \\
\hline Serotype $11 \mathrm{~A}$ & 0 & 0 & 1 & 1 & 4 & 3 & 3 & 5 & 2 & 4 & 23 & 12 & 52.2 \\
\hline Serotype 3 & 0 & 1 & 3 & 0 & 3 & 4 & 1 & 2 & 4 & 3 & 21 & 3 & $14 \cdot 3$ \\
\hline Serotype 1 & 0 & 0 & 1 & 2 & 3 & 5 & 1 & 1 & 1 & 0 & 14 & 1 & 7.1 \\
\hline Serotype 13 & 0 & 2 & 0 & 1 & 6 & 1 & 0 & 0 & 1 & 0 & 11 & 0 & 0.0 \\
\hline All other types ${ }^{a}$ & 8 & 5 & 5 & 9 & 16 & 17 & 15 & 10 & 13 & 23 & 121 & 38 & 31.4 \\
\hline Total & 58 & 150 & 185 & 207 & 268 & 210 & 120 & 108 & 97 & 133 & 1,536 & 896 & 58.3 \\
\hline $\begin{array}{l}15 \mathrm{~A} \text { as } \% \text { typed } \\
\text { reference submissions }{ }^{\mathrm{b}}\end{array}$ & 0.0 & 2.7 & 1.6 & 3.9 & 8.6 & \begin{tabular}{|l|}
10.8 \\
\end{tabular} & $14 \cdot 3$ & $24 \cdot 3$ & 33.7 & 29.0 & 11.0 & & \\
\hline
\end{tabular}

$95 \%$ of isolates were from England, Wales and Northern Ireland with the remainder from Scotland, Crown Dependencies, Republic of Ireland or elsewhere.

a Not accounting for $>10$ isolates in total over the surveillance period.

b Excludes 'no data row' above from denominator.

(mostly in England and Wales) consider to be concerning. Overall, 896/1,536 (58.3\%) had triple resistance. In the earlier years members of serotypes $19 \mathrm{~F}, 9 \mathrm{~V}, 6 \mathrm{~B}$ and 14 dominated, collectively accounting for $82.8-92.4 \%$ of referrals from 2005 to 2007 , before declining from the start of the 'PCV7 era'. Serotype $19 \mathrm{~A}$ accounted for a growing proportion of referrals from 2005 , peaking at $23.3 \%$ in 2011 , while serotype $15 \mathrm{~A}$ represented just $0-4 \%$ of submissions throughout the period 2005 to 2008 but thereafter increased progressively, becoming the most commonly referred serotype in 2012. In 2013 , it accounted for $31 / 92$ of all submissions where typing was undertaken, and for 31/107 in 2014. These proportions were greater than ever previously achieved by any other serotype. Fully $83.0 \%$ of serotype $15 \mathrm{~A}$ isolates (137/165) had the triple resistance vs 68.9-80.0\% among serotype $9 \mathrm{~V}, 19 \mathrm{~A}$ and $19 \mathrm{~F}$ referrals, with lower proportions for other serotypes (Table 4).

\section{Genomic sequencing and phenotypes of serotype $15 \mathrm{~A}$ isolates}

Genomic sequencing was performed on 156 serotype 15A S. pneumoniae. These represented a diversity of resistance patterns, and including 50 with triple resistance; a limitation was that all 156 sequenced isolates dated from 2013 and 2014. MLST types were deduced from the sequence data, and 78 (50\%) of the isolates were identified as belonging to ST63 $(n=61)$ or its single or double locus variants $(n=17)$. All of these 78 ST63-related isolates were resistant to erythromycin (also clindamycin, not shown) and 49 (62.8\%) had the triple resistance profile (Table 6). The macrolide and clindamycin resistance correlated with the consistent presence of $\operatorname{erm}(B)$ genes, as detected by WGS. All 78 ST63-related isolates were found also to carry the tetracycline-resistance determinant, $\operatorname{tet}(M)$; those $(n=65$, $83.3 \%$ ) that expressed tetracycline resistance had the intact gene, whereas those $(n=13,16.7 \%)$ that were tetracycline-susceptible (all of them classical ST63 isolates) had a deletion of two nucleotides at codon 339, generating a premature stop codon and thereby inactivating the gene. Most of the 49 isolates with triple resistance were susceptible to alternative agents: 37 remained susceptible to ampicillin, 47 to moxifloxacin, 48 to cefotaxime and all 49 to vancomycin, all based on EUCAST breakpoints. Sequence types (STs) 3811 $(n=19), 58$ and its single locus variants (SLVs) $(n=21)$, and 73 and its SLVs $(n=11)$ were all heavily represented among the 78 ST63-unrelated serotype $15 \mathrm{~A}$ isolates and, among all these, just one isolate had triple resistance and three or fewer were non-susceptible to any one of erythromycin, tetracycline or penicillin.

WGS data were available for a further 141 non-15A S. pneumoniae, predominantly investigated owing to multidrug resistance. Six had ST63-related profiles and 
Sequence types in relation to resistance of serotype 15A Streptococcus pneumoniae subjected to genomic sequencing $(\mathrm{n}=156)$

\begin{tabular}{|l|c|c|c|c|c|}
\hline & \multicolumn{5}{|c|}{ Number (\%) non-susceptible (intermediate or resistant) } \\
\hline ST63 & $\mathrm{n}$ & Erythromycin & Tetracycline & Penicillin & Triple resistance \\
\hline ST63 SLV and DLV & 61 & $61(100 \%)$ & $48(78.7 \%)$ & $46(75.4 \%)$ & $35(57.4 \%)$ \\
\hline Other 15A $\mathrm{A}^{\mathrm{a}}$ & 17 & $17(100 \%)$ & $17(100 \%)$ & $14(82.4 \%)$ & $14(82.4 \%)$ \\
\hline
\end{tabular}

SLV: single locus variant; ST: sequence type.

a Includes 21 ST58 and SLVs, 19 ST3811, 11 ST73 and SLVs and 27 isolates belonging to sequence types with four representatives or fewer.

these all had triple resistance; three expressed serotype $19 \mathrm{~F}$, one serotype 21 and one $23 \mathrm{~F}$; the final isolate was typed using antisera as serotype 20 but was predicted to be serotype $11 \mathrm{~A}$ based on WGS; review suggests that the original serotype determination was in error. The association with $19 \mathrm{~F}$ (a $\mathrm{PCV}_{7}$ serotype) is notable (see Discussion), but members of this serotype were highly variable in terms of ST; among a total of 25 serotype $19 \mathrm{~F}$ isolates sequenced, 22 with triple resistance, we recorded 12 different known STs, along with two new variants. No single ST had more than four representatives.

\section{Discussion}

Deployment of PCVs has had clear public health benefits. The incidence of invasive pneumococcal disease has been reduced not only in vaccinated children, but also in elderly adults, who benefit from herd immunity [31]. There is also evidence of impact on noninvasive disease: thus, $\mathrm{PCV}_{7}$ deployment in the UK in 2006 also was followed by a $19 \%$ reduction in hospital admissions for community-acquired pneumonia (CAP) among children aged $<2$ years, reversing a rising trend that had persisted during the preceding decade [32]. A similar reduction was reported in Italy [33]. Moreover, a Cochrane review concluded that $\mathrm{PCV}_{7}$ reduced the incidence of acute otitis media in healthy vaccinated children, although with less impact for those with a history of the illness or deemed to be 'high risk' [34]. Lastly, active PCV13 vaccination was recently shown to achieve a $50 \%$ reduction in the incidence of bacteraemia and non-invasive pneumonia in elderly adults, again reflecting displacement of vaccine serotypes [35].

A limitation to this pattern of successes is, however, that the PCV vaccines cover only the most prevalent pneumococcal serotypes, leaving scope for expansion of other types. Deployment of $\mathrm{PCV}_{7}$ was followed by increased prevalence of serotype $19 \mathrm{~A}$ isolates, many of them multidrug-resistant, and, although serotype $19 \mathrm{~A}$ is now covered by $\mathrm{PCV}_{13}$, a niche may be created for yet further types. Internationally, several groups have remarked on the increased prevalence of multidrug-resistant serotype $15 \mathrm{~A}$ and $35 \mathrm{~B}$ isolates [14-21] and a recent PHE analysis of invasive pneumococcal infections, using the data series of Table 3 , noted $15 \mathrm{~A}$ to be among several serotypes now increasing in numbers and proportion in the UK [13]. The present analysis extends these findings, confirming that serotype 15A S. pneumoniae are of growing importance, as also shown (i) in the BSAC bacteraemia series (Table 1), which overlaps the PHE series but also includes Scotland and Ireland, (ii) the BSAC series LRTI (Table 2), which is the sole UK surveillance to test $S$. pneumoniae from their predominant disease setting, and (iii) among PHE reference submissions, which provide a rolling snapshot of resistance phenotypes causing concern to microbiologists at sending laboratories, which are predominantly in England, Wales and Northern Ireland, although with a few isolates received from elsewhere (Table 5). By 2013 and 2014, serotype 15A was consistently (i) among the top 10 serotypes in both the PHE and BSAC surveillances of invasive S. pneumoniae (Tables 1 and 3), (ii) was the top serotype among respiratory isolates (Table 2) and (iii) accounted for almost one third of all the $S$. pneumoniae sent for reference investigation as 'unusually' resistant. Critically, and unlike other rising pneumococcal serotypes $(8,10 \mathrm{~A}, 11 \mathrm{~A}, 12 \mathrm{~F}$, and $24 \mathrm{~F}-$ see Tables 1, 3 and ref [13]) serotype $15 A$ isolates were commonly resistant or non-susceptible to multiple antibiotics, including macrolides, clindamycin, tetracycline and penicillin. While none of the surveillances captures clinical outcomes, the fact that serotype $15 \mathrm{~A}$ is rising in invasive infections implies that these organisms are virulent.

Around one third of serotype $15 \mathrm{~A}$ isolates had 'triple resistance' (i.e. to macrolides and tetracycline together with intermediate penicillin resistance), a higher proportion than for other serotypes (Table 4). This proportion did not change substantially over time (although assessment is complicated by small total numbers of isolates in the earlier years), indicating that the serotype was gaining prominence both generally and as a resistant type, again implying that the surface polysaccharides of serotype $15 \mathrm{~A}$ support virulence.

Triple resistance among serotype $15 \mathrm{~A}$ isolates was strongly associated ( $p<0.0001$, Fisher's exact of chisquared tests) with ST63 and its variants and extremely rare among serotype $15 \mathrm{~A}$ isolates belonging to other 
STs. This association between serotype 15A, ST63 and multidrug-resistance has been made by others too $[18,36,37]$ and it was suggested by Frazao et al. [38] that multidrug-resistant ST63-15A organisms arose by type transformation of ST63 strains previously expressing the $19 \mathrm{~F}$ capsular serotype. The present results provide very little support for this hypothesis. Although $3 / 22$ multidrug-resistant serotype $19 \mathrm{~F} \mathrm{~S}$. pneumoniae examined were ST63 single- or double-locus variants, the remaining $19 / 22$ belonged to diverse sequence types; moreover, ST63 alleles have been reported to be associated with other serotypes besides $19 \mathrm{~F}$ and $15 A$, including serotype 8 in Spain [39], where it is suggested that they may have arisen via serotype switching of earlier Sweden 15A lineages [40].

In summary, the present findings imply that conjugate vaccines will face an ongoing game of 'catch-up', as new serotypes rise to prominence, and that expansion beyond a 13-valent formulation will be needed. They are pertinent also to the debate as to whether PCV13 should be adopted for prophylactic vaccination against pneumonia in the elderly, as is advocated based on recent positive trial results in the Netherlands [35]. Such positive findings must be set against the fact that PCV13 strains now account for less than $20 \%$ of community-onset pneumococcal pneumonias in the UK (Table 2).

While the rise of any new multidrug-resistant type is of concern for patient management, the ST63-15A S. pneumoniae had high level resistances only to macrolides, clindamycin and tetracyclines; MICs of penicillin mostly remained in the range 0.12 to $0.5 \mathrm{mg} / \mathrm{L}$, and this level of 'non-susceptibility' is unlikely to compromise outcomes, except in meningitis. Susceptibility to moxifloxacin and cefotaxime remained near-universal, and ampicillin MICs were twofold below those of penicillin, remaining in the susceptible range and reversing the usual pattern for penicillin-non-susceptible S. pneumoniae, where ampicillin MICs mostly exceed those of penicillin. Treatment of infections therefore is unlikely to present especial problems, unless macrolides or tetracyclines are used alone, for example in beta-lactam allergic patients.

\section{Acknowledgements}

A considerable number of people have contributed to the success of this study. We are grateful to Dr Elizabeth Miller of Public Health England's (PHE) Immunisation Division, and to the members of the British Society for Antimicrobial Chemotherapy's Resistance Surveillance Standing Committee for helpful discussion. We are also grateful to Tony McNiff for help in data extraction and analysis and to the many PHE staff who undertook laboratory testing of these isolates, also to the NHS and Irish laboratories that have contributed isolates and data to the various surveillance programmes used here. This publication made use of the Streptococcus pneumoniae MLST website (http://pubmlst.org/spneumoniae/) sited at the University of Oxford [29]. The development of this site has been funded by the Wellcome Trust.

\section{Conflict of interest}

DML has shares in Pfizer and GSK, who make pneumococcal conjugate vaccines, and occasionally lectures and does contract and consultancy work for both companies. Other authors declare no conflict of interest.

\section{Authors' contributions}

CS, MK: molecular characterisation of isolates; NF/TH: Public Health England reference surveillance and typing of $\mathrm{S}$. pneumoniae, on which this analysis is predicated; RR/ SM/RJ: British Society for Antimicrobial Chemotherapy surveillance of $\mathrm{S}$. pneumoniae, on which this analysis is predicated; RP, RH, NW: reference investigation of resistant S. pneumoniae on which analysis is predicated; PS: extraction and consolidation of data series; MD: Bioinformatic analysis of sequence data; DML: primary observation of rise of $15 \mathrm{~A}$ S. pneumoniae, wrote manuscript. All authors commented upon and contributed to improving the manuscript.

\section{References}

1. Isaacman DJ, Fletcher MA, Fritzell B, Ciuryla V, Schranz J. Indirect effects associated with widespread vaccination of infants with heptavalent pneumococcal conjugate vaccine (PCV7; Prevnar).Vaccine. 2007;25(13):2420-7. DOI: 10.1016/j. vaccine.2006.09.011 PMID: 17049677

2. Rodrigo C, Bewick T, Sheppard C, Greenwood S, Macgregor V, Trotter $C$, et al. Pneumococcal serotypes in adult non-invasive and invasive pneumonia in relation to child contact and child vaccination status. Thorax. 2014;69(2):168-73. DOI: 10.1136/ thoraxjnl-2013-203987 PMID: 24048505

3. Myint TT, Madhava H, Balmer P, Christopoulou D, Attal S, Menegas D, et al. The impact of 7 -valent pneumococcal conjugate vaccine on invasive pneumococcal disease: a literature review. Adv Ther. 2013;30(2):127-51. DOI: 10.1007/ S12325-013-0007-6 PMID: 23397399

4. Isaacman DJ, Strutton DR, Kalpas EA, Horowicz-Mehler N, Stern LS, Casciano R, et al. The impact of indirect (herd) protection on the cost-effectiveness of pneumococcal conjugate vaccine. Clin Ther. 2008;30(2):341-57. DOI: 10.1016/j. clinthera.2008.02.003 PMID: 18343273

5. Song JH, Dagan R, Klugman KP, Fritzell B. The relationship between pneumococcal serotypes and antibiotic resistance.Vaccine. 2012;30(17):2728-37. DOI: 10.1016/j. vaccine.2012.01.091 PMID: 22330126

6. Dagan R, Klugman KP. Impact of conjugate pneumococcal vaccines on antibiotic resistance.Lancet Infect Dis. 2008;8(12):785-95. DOI: 10.1016/S1473-3099(08)70281-0 PMID: 19022193

7. Miller E, Andrews NJ, Waight PA, Slack MP, George RC. Herd immunity and serotype replacement 4 years after seven-valent pneumococcal conjugate vaccination in England and Wales: an observational cohort study. Lancet Infect Dis. 2011;11(10):7608. DOI: 10.1016/S1473-3099(11)70090-1 PMID: 21621466

8. Henderson KL, Muller-Pebody B, Blackburn RM, Johnson AP. Reduction in erythromycin resistance in invasive pneumococci from young children in England and Wales.J Antimicrob Chemother. 2010;65(2):369-70. DOI: 10.1093/jac/dkp442 PMID: 20007730

9. Clarke SC, Scott KJ, McChlery SM. Erythromycin resistance in invasive serotype 14 pneumococci is highly related to clonal type.J Med Microbiol. 2004;53(Pt 11):1101-3. DOI: 10.1099/ jmm.0.45737-o PMID: 15496387

10. Reinert R, Jacobs MR, Kaplan SL. Pneumococcal disease caused by serotype 19A: review of the literature and implications for future vaccine development.Vaccine. 2010;28(26):4249-59. DOI: 10.1016/j.vaccine.2010.04.020 PMID: 20416266

11. Liñares J, Ardanuy C, Pallares R, Fenoll A. Changes in antimicrobial resistance, serotypes and genotypes in Streptococcus pneumoniae over a 30-year period.Clin Microbiol Infect. 2010;16(5):402-10. DOI: 10.1111/j.14690691.2010.03182.x PMID: 20132251

12. van Hoek AJ, Sheppard CL, Andrews NJ, Waight PA, Slack MP, Harrison TG, et al. Pneumococcal carriage in children and adults two years after introduction of the thirteen valent pneumococcal conjugate vaccine in England. Vaccine. 2014;32(34):4349-55. DOI: 10.1016/j.vaccine.2014.03.017 PMID: 24657717 
13. Waight PA, Andrews NJ, Ladhani SN, Sheppard CL, Slack MP, Miller E. Effect of the 13-valent pneumococcal conjugate vaccine on invasive pneumococcal disease in England and Wales 4 years after its introduction: an observational cohort study. Lancet Infect Dis. 2015;15(5):535-43. DOI: 10.1016/S14733099(15)70044-7 PMID: 25801458

14. Ozawa D, Yano H, Endo S, Hidaka H, Kakuta R, Okitsu N, et al. Impact of the Seven-valent Pneumococcal Conjugate Vaccine on Acute Otitis Media in Japanese Children: Emergence of Serotype 15A Multidrug-resistant Streptococcus pneumoniae in Middle Ear Fluid Isolates. Pediatr Infect Dis J. 2015;34(9):e21721. DOI: 10.1097/INF.0000000000000776 PMID: 26083590

15. Suga S, Chang B, Asada K, Akeda H, Nishi J, Okada K, et al. Nationwide population-based surveillance of invasive pneumococcal disease in Japanese children: Effects of the seven-valent pneumococcal conjugate vaccine. Vaccine. 2015;33(45):6054-60. DOI: 10.1016/j.vaccine.2015.07.069 PMID: 26235372

16. Ho PL, Chiu SS, Law PY, Chan EL, Lai EL, Chow KH. Increase in the nasopharyngeal carriage of non-vaccine serogroup 15 Streptococcus pneumoniae after introduction of children pneumococcal conjugate vaccination in Hong Kong.Diagn Microbiol Infect Dis. 2015;81(2):145-8. DOI: 10.1016/j. diagmicrobio.2014.11.006 PMID: 25483278

17. Richter SS, Diekema DJ, Heilmann KP, Dohrn CL, Riahi F, Doern GV. Changes in pneumococcal serotypes and antimicrobial resistance after introduction of the 13 -valent conjugate vaccine in the United States.Antimicrob Agents Chemother. 2014;58(11):6484-9. DOI: 10.1128/AAC.03344-14 PMID: 25136018

18. Golden AR, Adam HJ, Gilmour MW, Baxter MR, Martin I, Nichol $\mathrm{KA}$, et al. Assessment of multidrug resistance, clonality and virulence in non-PCV-13 Streptococcus pneumoniae serotypes in Canada, 2011-13. J Antimicrob Chemother. 2015;70(7):19604.PMID: 25761605

19. Steens A, Bergsaker MA, Aaberge IS, Rønning K, Vestrheim DF. Prompt effect of replacing the 7-valent pneumococcal conjugate vaccine with the 13-valent vaccine on the epidemiology of invasive pneumococcal disease in Norway.Vaccine. 2013;31(52):6232-8. DOI: 10.1016/j. vaccine.2013.10.032 PMID: 24176490

20. Mameli C, Fabiano V, Daprai L, Bedogni G, Faccini M, Garlaschi $M L$, et al. A longitudinal study of streptococcus pneumoniae carriage in healthy children in the 13-valent pneumococcal conjugate vaccine era. Hum Vaccin Immunother. 2015;11(4):8117. DOI: $10.1080 / 21645515.2015 .1010945$ PMID: 25751237

21. Strachan JE, Rowe SL, Dunne EM, Hogg GG. Emergence of Streptococcus pneumoniae serotype $15 \mathrm{~A}$ after the introduction of the conjugate vaccine in Victoria.Med J Aust. 2013;199(7):461-3. DOI: 10.5694/mja13.10420 PMID: 24099202

22. British Society for Antimicrobial Chemotherapy (BSAC) Resistance Surveillance Project. [Accessed 15 July 2016] Available from: http://www.bsacsurv.org/

23. Reynolds R, Hope R, Williams L, BSAC Working Parties on Resistance Surveillance. Survey, laboratory and statistical methods for the BSAC Resistance Surveillance Programmes.J Antimicrob Chemother. 2008;62(Suppl 2):ii15-28. DOI: 10.1093/ jac/dkn349 PMID: 18819976

24. Farrell DJ, Felmingham D, Shackcloth J, Williams L, Maher $\mathrm{K}$, Hope R, et al. , BSAC Working Parties on Resistance Surveillance. Non-susceptibility trends and serotype distributions among Streptococcus pneumoniae from community-acquired respiratory tract infections and from bacteraemias in the UK and Ireland, 1999 to 2007.J Antimicrob Chemother. 2008;62(Suppl 2):ii87-95. DOI: 10.1093/jac/dkn355 PMID: 18819983

25. European Antimicrobial Resistance Surveillance Network (EARS-Net). [Accessed 15 July 2016]. Available from: http:// ecdc.europa.eu/en/healthtopics/antimicrobial-resistanceand-consumption/antimicrobial_resistance/EARS-Net/Pages/ EARS-Net.aspx

26. Lund E, Henrichsen J. Laboratory diagnosis, serology and epidemiology of Streptococcus pneumoniae. In: T. Bergan and J. R. Norris, editors. Methods in Microbiology. London: Academic Press; 1978. p. 241-262.

27. [No authors listed]. A guide to sensitivity testing. Report of the Working Party on Antibiotic Sensitivity Testing of the British Society for Antimicrobial Chemotherapy. J Antimicrob Chemother 1991;27 Suppl D:1-50.

28. The European Committee on Antimicrobial Susceptibility Testing - EUCAST. [Accessed Accessed 15 July 2016]. Available from: http://www.eucast.org/

29. Public databases for molecular typing and microbial genome diversity (PubMLST). Streptococcus pneumonia MLST Databases. Oxford: Department of Zoology, University of Oxford. [Accessed 27 Feb 2016]. Available from: http:// pubmlst.org/spneumoniae/
30. Inouye M, Conway TC, Zobel J, Holt KE. Short read sequence typing (SRST): multi-locus sequence types from short reads. BMC Genomics. 2012;13(1):338. DOI: 10.1186/1471-2164-13-338 PMID: 22827703

31. Arguedas A, Soley C, Abdelnour A. Prevenar experience. Vaccine. 2011;29(Suppl 3):C26-34. DOI: 10.1016/j. vaccine.2011.06.104 PMID: 21896350

32. Koshy E, Murray J, Bottle A, Sharland M, Saxena S. Impact of the seven-valent pneumococcal conjugate vaccination ( $\left.\mathrm{PCV}_{7}\right)$ programme on childhood hospital admissions for bacterial pneumonia and empyema in England: national time-trends study, 1997-2008.Thorax. 2010;65(9):770-4. DOI: 10.1136/ thx.2010.137802 PMID: 20805169

33. Ansaldi F, Sticchi L, Durando P, Carloni R, Oreste P, Vercelli $M$, et al. Decline in pneumonia and acute otitis media after the introduction of childhood pneumococcal vaccination in Liguria, Italy. J Int Med Res. 2008;36(6):1255-60. DOI: 10.1177/147323000803600612 PMID: 19094434

34. Fortanier AC, Venekamp RP, Boonacker CW, Hak E, Schilder AG, Sanders EA, et al. Pneumococcal conjugate vaccines for preventing otitis media. Cochrane Database Syst Rev. 2014;4(4):CDo01480.PMID: 24696098

35. Bonten MJ, Huijts SM, Bolkenbaas M, Webber C, Patterson S, Gault S, et al. Polysaccharide conjugate vaccine against pneumococcal pneumonia in adults. N Engl J Med. 2015;372(12):1114-25. DOI: 10.1056/NEJMoa1408544 PMID: 25785969

36. Naito S, Tanaka J, Nagashima K, Chang B, Hishiki H, Takahashi $Y$, et al. The impact of heptavalent pneumococcal conjugate vaccine on the incidence of childhood community-acquired pneumonia and bacteriologically confirmed pneumococcal pneumonia in Japan. Epidemiol Infect. 2016;144(3):494-506 DOI: $10.1017 /$ So950268815001272 PMID: 26122538

37. van der Linden $M$, Perniciaro S, Imöhl M. Increase of serotypes ${ }_{15} A$ and $23 B$ in IPD in Germany in the PCV13 vaccination era. BMC Infect Dis. 2015;15(1):207. DOI: 10.1186/s12879-015-09419 PMID: 25940580

38. Frazão N, Hiller NL, Powell E, Earl J, Ahmed A, Sá-Leão R, et al. Virulence potential and genome-wide characterization of drug resistant Streptococcus pneumoniae clones selected in vivo by the 7 -valent pneumococcal conjugate vaccine. PLoS One. 2013;8(9):e74867. DOI: 10.1371/journal.pone.0074867 PMID: 24069360

39. Ardanuy C, de la Campa AG, García E, Fenoll A, Calatayud L, Cercenado E, et al. Spread of Streptococcus pneumoniae serotype 8-ST63 multidrug-resistant recombinant Clone, Spain. Emerg Infect Dis. 2014;20(11):1848-56. DOI: 10.3201/ eid2011.131215 PMID: 25340616

40. Sanz JC, Cercenado E, Marín M, Ramos B, Ardanuy C, Rodríguez-Avial I, et al. Multidrug-resistant pneumococci (serotype 8) causing invasive disease in HIV+ patients. Clin Microbiol Infect. 2011;17(7):1094-8. DOI: 10.1111/j.14690691.2011.03495.x PMID: 21463396

\section{License and copyright}

This is an open-access article distributed under the terms of the Creative Commons Attribution (CC BY 4.0) Licence. You may share and adapt the material, but must give appropriate credit to the source, provide a link to the licence, and indicate if changes were made.

This article is copyright of the authors, 2016. 